\title{
Validation of the Arabic version of the Short Form Leed's Dyspepsia Index (SF-LDQ) amongst patients with type II diabetes
}

\author{
Khaled Alabdulkareem ${ }^{\mathrm{a}}$, Quratulain Shaikh ${ }^{\mathrm{b}, *}$, Sultanah Hisham Alsulaiman ${ }^{\mathrm{c}}$, Marvi Mahesar ${ }^{\mathrm{b}}$, \\ Nouf Marzuq Almutairi ${ }^{\mathrm{d}}$, Abdulaziz S. AlEissa ${ }^{\mathrm{d}}$, Sarah Saud AlWadaani ${ }^{\mathrm{e}}$, \\ Waad Mayouf Alanazi ${ }^{\mathrm{e}}$, Fatima Fadhel AlAmri ${ }^{\mathrm{e}}$ \\ ${ }^{a}$ Family Medicine at College of Medicine, Al Imam Mohammad Ibn Saud Islamic University (IMSIU), Othman Ibn Affan Street, 11432, Riyadh, Saudi Arabia \\ ${ }^{\mathrm{b}}$ Indus Hospital Research Center, The Indus Hospital, Plot C-76, Sector 31/5, Opposite Darussalam Society, Korangi Crossing, Karachi, 75190, Pakistan \\ ${ }^{\mathrm{c}}$ Health Sciences Research Center (HSRC), Princess Noura Bint Abdulrahman University, King Khalid International Airport, Riyadh, 13412, Saudi Arabia \\ ${ }^{\mathrm{d}}$ Family Medicine, King Abdullah Bin Abdulaziz University Hospital, King Khalid International Airport, Riyadh, 13412, Saudi Arabia \\ ${ }^{\mathrm{e}}$ King Abdullah Bin Abdulaziz University Hospital, King Khalid International Airport, Riyadh, 13412, Saudi Arabia
}

\section{A R T I C L E I N F O}

\section{Keywords:}

Diabetes mellitus type II

Dyspepsia

Validation study

\begin{abstract}
A B S T R A C T
Since an unambiguous clinical definition of dyspepsia does not currently exist, the diagnosis is made on the basis of symptoms. For this purpose, a tool which is valid, reliable and convenient to use is needed that can accurately assess both frequency and severity of dyspepsia. Short-Form Leeds Dyspepsia Questionnaire meets all of these requirements and thus, has to be validated in native languages before it can be used to diagnose dyspepsia. We conducted a cross-sectional study at King Abdullah Bin Abdulaziz University Hospital (KAAUH) in Riyadh, Saudi Arabia. Adult patients with type II diabetes were recruited in the study after giving informed consent. A total of 245 patients were recruited in the study; 95 (38.8\%) were males, while $149(61.1 \%)$ were females. We found that $27.3 \%(n=67)$ of the participants had dyspepsia according to the SF-LDQ Arabic version compared to $24.1 \%$ ( $n$ = 59) by physician's diagnosis. The Area Under the Curve was 0.86 (95\% CI: 0.81-0.9). At a cut-off value of 7/ 32 , the tool has a sensitivity of $69.49 \%$ and a specificity of $83.24 \%$. In conclusion, The Short-Form Leeds Dyspepsia Questionnaire was found to be valid for assessment of frequency and severity of dyspepsia among Arab diabetes Type 2 patients.
\end{abstract}

\section{What we already know}

- Dyspepsia is a common symptom amongst patients with Type II Diabetes Mellitus

- Since there is no clinically established definition of dyspepsia, the diagnosis is made on the basis clinical history and examination

- The Short Form - Leeds Dyspepsia Questionnaire (SF-LDQ) is a validated instrument that assesses both frequency and severity. Therefore, it provides an accurate measure of clinical diagnosis

\section{What this article adds}

- Validates the Short Form - Leeds Dyspepsia Questionnaire (SF-LDQ) in the Arabic language
- Determines the prevalence of dyspepsia, on the basis of severity and frequency, amongst the Arab population with Type II Diabetes Mellitus

- Establishes the diagnostic accuracy of the tool, determining both its sensitivity and specificity

\section{Introduction}

Although dyspepsia is a commonly used term in clinical practice, there is a significant amount of ambiguity regarding its definition. ${ }^{1}$ One of the commonly accepted definitions of dyspepsia is, presence of pain, discomfort or nausea relating to upper gastrointestinal tract, which does not result from physical strain, nor resolves within $5 \mathrm{~min}$ of rest, and is either continuous or intermittent. ${ }^{2}$ Dyspepsia is considered to be a global

\footnotetext{
* Corresponding author.

E-mail addresses: kialabdulkareem@imamu.edu.sa (K. Alabdulkareem), quratulain.shaikh@tih.org.pk (Q. Shaikh), sultana.alsulaiman@gmail.com

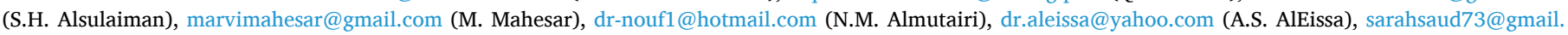
com (S.S. AlWadaani),wm15a2@hotmail.com (W.M. Alanazi), fattoo80@hotmail.com (F.F. AlAmri). 
health issue, with a gross prevalence of $25 \% .{ }^{3}$ In patients with Type II diabetes, gastrointestinal symptoms, ranging from mild dyspepsia to gastroparesis, are fairly common. ${ }^{4}$ Gulcelik et al. conducted a study, in which $60.3 \%$ of the diabetic patients exhibited symptoms of dyspepsia. ${ }^{5}$ Another study published by Osipenko et al., in 2013 found that the prevalence of dyspepsia amongst Type II diabetics was $71 \%{ }^{6}$

According to a systematic review of 21 peer reviewed articles published in 2016, the prevalence of type II diabetes mellitus in Saudi Arabia was $32.8 \%$. The predicted prevalence of this disease was estimated to rise to $35.37 \%$ in 2020 and $45.36 \%$ in 2030 .

Due to the absence of a clinically established definition of dyspepsia, the diagnosis is made on the basis clinical history and examination sometimes aided by validated tools. ${ }^{8}$ Uni-dimensional questionnaires measure either the frequency or severity of dyspepsia; it has been determined that frequency is more closely associated with a clinical diagnosis of dyspepsia, while severity is a better indicator of response to therapy. ${ }^{9}$ The Short Form - Leeds Dyspepsia Questionnaire (SF-LDQ) is a validated instrument, which was designed in 2007, that assesses both frequency and severity, therefore, providing an accurate measure of clinical diagnosis and responsiveness to change. ${ }^{10}$ The tool has been validated in numerous languages before, such as English, Italian and Kinyarwanda; this study aimed to use this instrument to measure frequency and severity of dyspepsia amongst type II diabetic patients and validate the tool in the Arabic language. ${ }^{10,11,12}$

\section{Materials and methods}

\subsection{SF-LDQ and translation}

SF-LDQ is a shortened version of the Leeds Dyspepsia Questionnaire (LDQ), a validated 8-item questionnaire assessing frequency and severity. This 4-item tool assesses, over the last two months, the frequency on the basis of symptoms of upper gastrointestinal tract, such as indigestion, heartburn, regurgitation and nausea, while severity is assessed by scoring the questionnaire from $0-32 .{ }^{13}$ The responses are scored from 0 (not at all) to 4 (once a day or more), the overall score is then calculated by summing the scores of frequency and severity of all symptoms. There is an additional question inquiring about the symptom which the patient finds the most inconvenient. ${ }^{13}$ SF-LDQ was translated into Arabic, independently, by two bilingual native speakers. It was then back translated into English and reviewed by two family physicians (native Arabs) for clarity and content validity.

\subsection{Patients}

All adult patients with type II diabetes coming to the Diabetes Clinic at King Abdullah Bin AbdulAziz University Hospital (KAAUH) in Riyadh, Saudi Arabia were approached to participate in the study. Those who gave their consent were included in the study.

\subsection{SF-LDQ evaluation}

After consent, patients were asked some questions on their demographic and clinical history and the Arabic version of SF-LDQ was self-administered before they went into the physician's office. The physician took detailed medical history and assessed all patients for symptoms of dyspepsia, blinded to their interview outside, so that the diagnosis could be confirmed clinically. This physician's assessment was taken as a "quasi gold standard" for comparing the performance of the SF-LDQ Arabic version. Routine laboratory parameters were also recorded; the most recent report of HbA1c was used to determine control of diabetes over the last 3 months. Data was analyzed using SPSS version 24. SF-LDQ scores were calculated by summing the individual frequency and severity scores for each symptom to produce a total summed score. The range of the total score was 0-32. A Receiver Operating Characteristics (ROC) curve was then plotted against the physician's diagnosis to determine the point of greatest specificity and sensitivity. A twotailed $P$-VALUE $<0.05$ was considered significant.

\section{Results}

Among the 245 participants, $61 \%$ were females (Table 1) and most had studied till bachelor's level (32.7\%). Majority of the participants $(76.7 \%)$ were married and $88.6 \%$ were Saudis. Median age of the participants was 55 (47-61) years. Mean age of male participants was 54.7 (12.1) years, with minimum and maximum age being 20 and 81 years, respectively. While, the mean age of female participants was 54 (13.6) years, with minimum and maximum age being 18 and 77 years, respectively. There was no significant difference in the duration or control of diabetes (HbA1C) when the genders were compared ( $P$ $V A L U E>0.05$ ) (Supplementary Table 1). Similarly, no relationship was observed between dyspepsia frequency (both on SF-LDQ and physician's assessment) and diabetes control ( $P$-VALUE $>0.05)$ (Supplementary Table 2).

\subsection{Dyspepsia frequency}

Cutoff scores for frequency and severity of dyspepsia were based on those outlined by an SF-LDQ validation study conducted by Fraser et al. ${ }^{13}$ On the basis of the cutoff for summed frequency score of dyspepsia $(4 / 16), 25.3 \%(n=62)$ of the 245 participants were categorized as having dyspepsia. On the basis of the cutoff for summed severity score of dyspepsia $(2 / 16), 30.2 \%(n=74)$ participants were categorized as having dyspepsia. On the basis of the cutoff for summed total score of both frequency and severity $(7 / 32), 27.3 \%(n=67)$ participants were classified as having dyspepsia on SF-LDQ Arabic version, whereas $72.7 \%$ ( $\mathrm{n}=178$ ) were classified as having no dyspepsia. According to the physician's diagnosis, $24.1 \%(n=59)$ participants from this population had dyspepsia on clinical examination, whereas $75.5 \%(n=185)$ did not have dyspepsia.

\subsection{Concurrent validity OF SF-LDQ (Arabic version)}

Concurrent validity was determined by comparing the physician's clinical diagnosis with the scores of SF-LDQ; this was seen in 244 participants who had both physician's diagnosis and SF-LDQ scores. It was seen that there was no significant difference between the frequency of dyspepsia on SF-LDQ (27.3\%) and physician's diagnosis $(24.1 \%)(P$ VALUE $>0.05$ : Table 2). When assessed by both methods $15 \%$ of the participants $(\mathrm{n}=37)$ had dyspepsia and 63.5\% $(\mathrm{n}=155)$ had no dyspepsia.

Table 1

Baseline characteristics of participants.

\begin{tabular}{llll}
\hline Demographics & Categories & Frequency & Percentage \\
\hline Gender & Male & 95 & 38.8 \\
Highest level of education & Female & 149 & 61.1 \\
& None at all & 9 & 3.7 \\
& Primary school & 18 & 7.3 \\
& Secondary school & 14 & 5.7 \\
& High school & 31 & 12.7 \\
Marital status & Bachelors & 80 & 32.7 \\
& Single & 27 & 11 \\
& Married & 188 & 76.7 \\
Nationality & Separated & 1 & 0.4 \\
& Divorced & 5 & 2 \\
Age & Widowed & 18 & 7.3 \\
& Saudi & 217 & 88.6 \\
& Non-saudi & 24 & 9.8 \\
& Mean (SD) & Min-Max & Median (IQR) \\
\hline
\end{tabular}


Table 2

Concurrent validity between SF-LDQ Arabic version \& physician's diagnosis.

\begin{tabular}{lllll}
\hline \multicolumn{4}{c}{ Physician's diagnosis } \\
\hline $\begin{array}{l}\text { SF-LDQ Summed total } \\
\text { dyspepsia score }{ }^{\mathrm{b}}\end{array}$ & Dyspepsia & No & Total & P- \\
Dyspepsia & & dyspepsia & & Value \\
No dyspepsia & $37(15.2)$ & $22(9)$ & $59(24.2)$ & $0.332^{\text {a }}$ \\
& $30(12.3)$ & $155(63.5)$ & 185 & \\
& & & $(75.8)$ & \\
\hline
\end{tabular}

${ }^{\text {a }}$ McNemar test applied.

b Cut-off score: $7 / 32$.

\subsection{Diagnostic accuracy}

A Receiver Operating Characteristics (ROC) curve was then plotted against the physician's diagnosis to determine the appropriate specificity and sensitivity (Fig. 1). Similar to the recommendation by Fraser et al. the cutoff point with the most sensitivity and specificity was $7 / 32$ in our data also. ${ }^{13}$ At this cut-off the sensitivity of SF-LDQ was found to be $69.49 \%$, while the specificity was $83.24 \%$ with the best correct classification rate of $79.9 \%$ and AUC of 0.86 (95\% CI: 0.81-0.9) (Supplementary Table 3).

\section{Discussion}

Currently, there is no established "gold standard" to diagnose dyspepsia. ${ }^{9,14}$ In this study, the physician's clinical diagnosis was used as a "quasi gold standard" to determine concurrent validity. There have been a number of studies in which the clinical diagnosis was used as a reference for comparison in the absence of any "gold standard". ${ }^{15-17}$ Other studies have used scores of quality of life questionnaires, ${ }^{18,19}$ written accounts of self-assessment by patients ${ }^{20}$ and severity of dyspepsia assessment as alternatives. ${ }^{21}$ In our study, using clinician's diagnosis for concurrent validity led to $69.49 \%$ sensitivity and $83.24 \%$ specificity of SF-LDQ. This indicates that the Arabic validated version of SF-LDQ can be used to screen for dyspepsia in the Arabic speaking population.

Dyspepsia is seen frequently in diabetics, probably related to the autonomic neuropathy, gastroparesis and a presumed relationship with $\mathrm{H}$. pylori bacteria. A study conducted in India reported an $\mathrm{H}$. pylori prevalence of $77 \%$ among diabetics compared to $58 \%$ in controls $(P$ VALUE $<0.05) .{ }^{22}$ However, other studies fail to show any association of

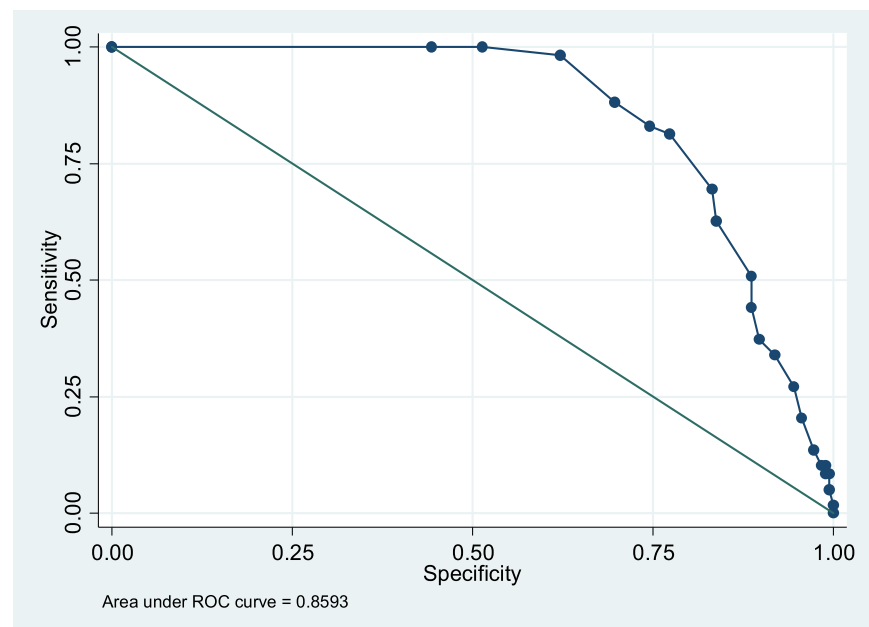

Fig. 1. Receiver Operating Characteristic (ROC) curve for the summed total score of the SF-LDQ Arabic version.

AUC $=0.86(95 \%$ CI:0.81-0.90).
H. pylori with diabetes mellitus. ${ }^{23-26}$ Due to the high prevalence of this condition in diabetics, we chose to validate the Arabic version of SF-LDQ in the native diabetic Arab population.

A number of tools have been developed to assess dyspepsia but majority are either too lengthy to be used in an outpatient setting or lack assessment of severity of symptoms. ${ }^{18,20,21}$ A systematic review of symptom based questionnaires for dyspepsia and GERD identified 26 questionnaires amongst which only 11 assessed both frequency and severity of symptoms and Leeds Dyspepsia Questionnaire was one of them. ${ }^{8}$ It's Short form (SF-LDQ) was developed in $2007^{13}$ and has since been translated in numerous languages. ${ }^{11,12,27,28}$

Saudi Arabia has a high diabetes prevalence which is projected to increase. ${ }^{7}$ This will lead to a huge segment of the population suffering from its associated conditions like dyspepsia requiring chronic management. For the assessment of dyspepsia, only one validated tool exists in Arabic language, the 'Nepean Dyspepsia Index' consisting of 42 items falling short of a quick screener in the outpatient setting. ${ }^{29}$ Hence, the current validated version of SF-LDQ, will serve as a screening tool allowing for early diagnosis and better assessment of response to therapy in the Arab population.

In the original study by Fraser et al. the recommended summed total cutoff score for diagnosis of dyspepsia was also $7 / 32$; with a sensitivity of $77.3 \%$ and specificity of $73.2 \% .{ }^{13}$ Another similar study, using the Italian version of SF-LDQ, found sensitivity of $80.4 \%$ and a specificity of 82.5\% for dyspepsia. ${ }^{11}$ However, these studies also measured discriminant validity along with test-retest reliability which should be the next step forward.

In conclusion, the Arabic version of SF-LDQ has proven to be a valid and feasible tool to accurately measure the frequency and severity of dyspepsia in the outpatient setting. We recommend testing the instrument for test re-test reliability and validation among other patient populations than diabetes mellitus type 2 .

\section{Conclusion}

Based on the results of our study, we conclude that SF-LDQ is both a sensitive and specific tool which can be used to determine the frequency and severity of dyspepsia. Arabic version of SF- will prove to be of immense value in conducting a quick clinical screen along with contributing to the determinants of dyspepsia in this population. We recommend further research and the validation of this Arabic version in other patient populations and age groups so that it can benefit further.

\section{Limitations}

Our study did not measure parameters such as discriminant validity, test-retest reliability and responsiveness to change since it was not feasible to follow up these patients. Measurement of these parameters would have added to the validation of the SF-LDQ questionnaire in the Arabic language and provided a more accurate assessment of the psychometric properties of the tool. We used a quasi-gold standard of physician diagnosis as a reference but we believe that dyspepsia is a symptom which can be present in the absence of any documented pathology (functional dyspepsia). Hence, it is not possible to select a perfect "gold standard" for comparison of the results. The original author 1 has also used physician diagnosis for validation of the tool. ${ }^{13}$

\section{Ethical statement}

Institutional Review Board of Princess Nourah Bint Abdulrahman University, IRB Registration Number with KACST, KSA: H-01-R-059, RB Log Number: 18-0001, Category of Approval: EXEMPT.

Study was EXEMPT from IRB Review as it posed no more than minimal risk to participants. Informed consent was taken from participants before being included in the study.

The paper reflects the authors' own research and analysis in a 
truthful and complete manner. All authors have been personally and actively involved in substantial work leading to the paper, and will take responsibility for its content.

\section{Declaration of competing interest}

The authors declare that they have no conflict of interest pertaining to this study.

\section{Appendix A. Supplementary data}

Supplementary data to this article can be found online at https://doi. org/10.1016/j.cegh.2021.100790.

\section{References}

1 Heading RC. Definitions of dyspepsia. Scand J Gastroenterol Suppl. 1991;182(sup182): 1-6.

2 Talley NJ, McNeil D, Piper DW. Discriminant value of dyspeptic symptoms: a study of the clinical presentation of 221 patients with dyspepsia of unknown cause, peptic ulceration, and cholelithiasis. Gut. 1987;28(1):40-46.

3 Del Valle J. Peptic ulcer disease and related disorders. Har Prin of Int med. 2005;16 (2):1746.

4 Xia HH-X, Talley NJ, Kam EP, Young LJ, Hammer J, Horowitz M. Helicobacter pylori infection is not associated with diabetes mellitus, nor with upper gastrointestinal symptoms in diabetes mellitus. Am J Gastroenterol. 2001;96(4):1039-1046.

5 Gulcelik NE, Kaya E, Demirbas B, et al. Helicobacter pylori prevalence in diabetic patients and its relationship with dyspepsia and autonomic neuropathy. $J$ Endocrinol Invest. 2005;28(3):214-217.

6 Osipenko MF, Zhuk EA, Medvedeva OV. [Clinical characteristics of dyspepsia in patients with diabetes mellitus type 2]. Ter Arkh. 2013;85(2):43-47.

7 Meo SA. Prevalence and future prediction of type 2 diabetes mellitus in the Kingdom of Saudi Arabia: a systematic review of published studies. J Pakistan Med Assoc. 2016; 66(6):722-725.

8 Fraser A, Delaney B, Moayyedi P. Symptom-based outcome measures for dyspepsia and GERD trials: a systematic review. Am J Gastroenterol. 2005;100(2):442-452.

9 Agreus L. Natural history of dyspepsia. Gut. 2002;50(suppl 4) (suppl 4):iv2-9.

10 Fraser A, Qume M, Ford A, et al. Validation of the short-form Leeds dyspepsia questionnaire (SF-LDQ) in a community setting. Gastroenterology. 2003;124(4):A224.

11 Gatta L, Moayyedi P, Tosetti C, et al. A validation study of the Italian short-form Leeds dyspepsia questionnaire. Intern Emerg Med. 2010;5(6):501-506.

12 Nkurunziza A, Dusabejambo V, Everhart K, Bensen S, Walker T. Validation of the Kinyarwanda-version short-form Leeds dyspepsia questionnaire and short-form nepean dyspepsia index to assess dyspepsia prevalence and quality-of-life impact in Rwanda. BMJ Open. 2016;6(6), e011018.
13 Fraser A, Delaney BC, Ford AC, Qume M, Moayyedi P. The short-form Leeds dyspepsia questionnaire validation study. Aliment Pharmacol Ther. 2007;25(4): $477-486$.

14 Dent J. Definitions of reflux disease and its separation from dyspepsia. Gut. 2002;50 (suppl 4) (suppl 4):iv17-20; discussion iv1-2.

15 Junghard O, Lauritsen K, Talley NJ, Wiklund IK. Validation of seven graded diary cards for severity of dyspeptic symptoms in patients with non ulcer dyspepsia. Eur $J$ Surg Suppl. 1998;(583):106-111.

16 Greatorex R, Thorpe JA. Clinical assessment of gastro-oesophageal reflux by questionnaire. Br J Clin Pract. 1983;37(4):133-135.

17 Buckley MJ, Scanlon C, McGurgan P, O'Morain CA. A validated dyspepsia symptom score. Ital J Gastroenterol Hepatol. 1997;29(6):495-500.

18 Hu WH, Lam KF, Wong YH, et al. The Hong Kong index of dyspepsia: a validated symptom severity questionnaire for patients with dyspepsia. J Gastroenterol Hepatol. 2002;17(5):545-551.

19 Dimenäs E, Glise H, Hallerbäck B, Hernqvist H, Svedlund J, Wiklund I. Quality of life in patients with upper gastrointestinal symptoms: an improved evaluation of treatment regimens? Scand J Gastroenterol Suppl. 1993;28(8):681-687.

20 Leidy NK, Farup C, Rentz AM, Ganoczy D, Koch KL. Patient-based assessment in dyspepsia: development and validation of dyspepsia symptom severity index (DSSI). Dig Dis Sci. 2000;45(6):1172-1179.

21 Rabeneck L, Wristers K, Goldstein JL, Eisen G, Dedhiya SD, Burke TA. Reliability, validity, and responsiveness of severity of dyspepsia assessment (SODA) in a randomized clinical trial of a COX-2-specific inhibitor and traditional NSAID therapy. Am J Gastroenterol. 2002;97(1):32-39.

22 Bajaj S, Rekwal L, Misra SP, Misra V, Yadav RK, Srivastava A. Association of helicobacter pylori infection with type 2 diabetes. Indian J Endocrinol Metab. 2014;18 (5):694-699.

23 Demir M, Gokturk HS, Ozturk NA, Kulaksizoglu M, Serin E, Yilmaz U. Helicobacter pylori prevalence in diabetes mellitus patients with dyspeptic symptoms and its relationship to glycemic control and late complications. Dig Dis Sci. 2008;53(10): 2646-2649.

24 Abboud A, Moussawi H, Rustom M, Khalek W. Epidemiology of Helicobacter pylori infection among symptomatic patients, correlation with endoscopic findings and it's association with type II diabetes mellitus. J Gastrointest Dig Syst. 2017;7(3):1-5.

25 Alipour Z, Khatib F, Tabib SM, et al. Assessment of the prevalence of diabetic gastroparesis and validation of gastric emptying scintigraphy for diagnosis. Mol Imaging Radionucl Ther. 2017;26(1):17-23.

26 Vaishnav BT, Shaikh SR, Bamanikar AA, Kakrani AL, Tambile RR. Diagnostic upper gastrointestinal endoscopy and prevalence of Helicobacter Pylori infection in dyspeptic type 2 diabetes mellitus patients. J Dig Endosc. 2018;9 (02):053-60.

27 Mahadeva S, Chan WK, Mohazmi M, Sujarita R, Goh KL. Validation study of the Leeds dyspepsia questionnaire in a multi-ethnic asian population. J Gastroentero Hepatol. 2011;26(11):1669-1676.

28 Validation study of the Indonesian short form-leeds dyspepsia questionnaire (SFLDQ)Prasetya IB, Anas D, Al-Idrus SNE, Simatupang FS, Herardi R, Budiyani L, eds. J Dig Endosc. 2018. WILEY 111 RIVER ST, HOBOKEN 07030-5774, NJ USA.

29 Khalil MS, Wahass SH, Al-Qourain AA, Yassawy MI. Initial linguistic and psychometric validation of the Arabic version of Nepean Dyspepsia Index. Saudi Med J. 2006;27(10):1554-1560. 